\title{
Long-Term Prediction of Vitreous Materials Degradation Derived from Short-Term Testing
}

\author{
A.A. Kruger
}

US Department of Energy, Office of River Protection, H6-60, Richland, WA 99352, USA

(Albert_A_Kruger@ORP.DOE.gov)

Aging and Durability of Ancient Glasses to

Predicted Long-Term Performance of Vitrified

Waste

As advanced by the conveners of this theme, "it is crucial to understand material corrosion as a function of environmental conditions (temperature, $\mathrm{pH}, \mathrm{Eh}$, and microbial activity, etc.) over centuries to millennia." This presents a significant challenge to practitioners in establishing a testing protocol that mimics the thermodynamic and kinetic controls of a long-term process in a procedure that is completed in a useful length of time (e.g., 5 days to a fortnight). The test procedure is best effectuated in a temperature regime that closely mimic those of the disposal facility. This while giving results that are fully representative of the wasteform degradation at the specific environmental conditions of the disposal site.

\section{Current Endeavors}

The concept of immobilizing radioactive waste in either vitreous or crystalline materials is more than 50 years old ${ }^{1}$. During 1970s, "low leachability" or high chemical durability had become the main criterion for waste form selection. Between 1979 - 1981, U.S. DOE conducted its National High-Level Waste Technology Program to develop and evaluate candidate waste forms for immobilization of HLW at DOE defense sites. A total of 17 waste forms were initially considered for development and evaluation. Based on waste form evaluations conducted at DOE national laboratories, independent laboratories, peer review assessments, product performance evaluations, and a processability assessment, Borosilicate glass was chosen as the reference waste form. Current leaching tests (e.g., Vapor Hydration Test - VHT) for immobilized Low-Activity waste do not reflect anticipated disposal conditions and do not provide comparative information for other waste forms. This current test exhibits high variability between labs, high variability between operators and is fraught with large uncertainties in dissolution rate. As such we have embarked on an endeavor to develop and validate leaching tests that reflect disposal conditions.

${ }^{1}$ Bao, Y M. W Grutzeck, C. M. Jantzen, J. Am. Ceram. Soc. 88 (2005) 3287 